\title{
Effects of Concentrated Growth Factor and Cervical Lymph Node Metastasis on the Prognosis of Mucoepidermoid Carcinoma of the Parotid Gland
}

\author{
Shang-Zhi Han, Xin Lv, Xi-Bo Chen, Ying-Ying Xu, Rui-Liu, Xing-Le Zhang \\ Department of Stomatology, the Affiliated Hospital of Chengde Medical College, Chengde, Hebei Province, PR China.
}

\begin{abstract}
Introduction: Mucoepidermoid carcinoma (MEC) is a common salivary gland malignancy. As studies reported on large cases of mucoepidermoid carcinoma of the parotid glands are few, this paper aims to research the effects of clinical and pathological factors, such as applying concentrated growth factor (CGF) on repairing and cervical lymph node metastasis, on the prognosis of mucoepidermoid carcinoma of the parotid gland.
\end{abstract}

Methods: The retrospective analysis of prognostic factors was conducted based on 176 cases with mucoepidermoid carcinoma of the parotid gland, who received treatment at the Affiliated Hospital of Chengde Medical College during the period of March 2000 to March 2012.

Results: The Five-year overall survival rate was $75.57 \%$, while the Five-year tumor-free survival rate was $64.77 \%$. Univariate analysis showed that the influential factors for the prognosis of mucoepidermoid carcinoma of the parotid gland included surgical approach, tumor size, clinical stage, pathological grade, lymph node metastasis, and distant metastasis, etc; among which pathological grade, lymph node metastasis, and distant metastasis were indicated by multivariate analysis to be the independent risk factors.

Conclusion: The survival rate of mucoepidermoid carcinoma of the parotid gland is relatively high. Lymph node metastasis, pathological grade, and distant metastasis are the independent risk factors that affect the prognosis of patients with mucous epidermoid carcinoma of the parotid gland.

Keywords: Mucoepidermoid Carcinoma, CGF, Lymph Node Metastasis, Prognostic Factors

\section{Introduction}

Mucoepidermoid carcinoma (MEC) is the most common malignant tumor in salivary glands, accounting for $3 \%$ $-15 \%$ of all salivary gland tumors, of which $62 \%$ occur in the parotid glands. 1 In recent years, the incidence of MEC of the parotid glands shows rising and younger trends. ${ }^{2}$ As researches on large cases of mucoepidermoid carcinoma of the parotid glands are rarely reported, this study retrospectively analyzed the clinicopathological data of 176 cases with parotid MEC, and explored the relevant factors affecting its prognosis.
Concentrated growth factor (CGF) is a gel-like mass containing high concentrations of various growth factors and fibrin isolated from autologous blood by centrifugation and has the function of promoting tissue regeneration. ${ }^{3}$ It can promote tissue healing and reduce postoperative scars. ${ }^{4}$

\section{Methods}

Clinical data: One hundred seventy-six (176) patients with parotid MEC who underwent surgical treatment at the Affiliated Hospital of Chengde Medical College from

Correspondence

Xing-Le Zhang, Department of Stomatology, the Affiliated Hospital of Chengde Medical College, Chengde, Hebei, China.email:shangzhihanhan@163.com 
March 2000 to March 2013 were collected, including 92 males and 84 females with the ratio of male to female 1: 1.10 and patients aged from 12 to 87 years with a median age of 45 years. The clinical staging was made according to the 2002 classification of the International Union Against Cancer (UICC 2002), and the diagnosis of MEC was made through clinical examination combined with magnetic resonance imaging (MRI) or computed tomography (CT) as follows: 29 patients presented with clinical stage I, 50 patients in stage II, 36 patients in stage III, and 61 patients in stage IV. Cervical lymphadenopathy was present in 53 patients by clinical physical examination and palpation, among which 25 patients were with lymph node metastasis. Concentrated growth factor (CGF) in the parotid gland was performed in 42 patients.

Inclusion criteria: 1: The patient with the primary lesion of MEC in the parotid gland, who got the first surgical treatment and non-recurrent cancer. 2: The patient preoperatively had no medical history of radiotherapy and chemotherapy. 3: The patient who had no other severe whole-body diseases. 4: The patient who had good compliance and complete case information.

Follow-up survey: The mean duration was from the first treatment till the latest follow-up in March, 2018 (median, 98 months; average, 110.28 months). Thirteen patients (7.4\%) were lost to follow up, and these censored data were processed from the date of losing follow-up. All the fatal cases had died of the primary disease.

Statistical methods: SPSS 16.0 software was used for statistical analysis. Kaplan-Meier method and Logrank test were used to evaluate the relationship between indicators and survival time, and Hazard Ratio (HR) and 95\% confidence interval ( $95 \% \mathrm{CI}$ ) were calculated by Cox proportional risk regression model. Cox multivariate regression analysis was performed for factors with $\mathrm{p}<0.1$ in univariate analysis. All tests were double-sided, and $\mathrm{p}<0.05$ was considered to be statistically significant.

\section{Results}

Survival rate: The Five-year overall survival rate was $75.57 \%$, while the Five-year tumor-free survival rate was $64.77 \%$. The median survival time was 81 months. As to the survival curve, Figure one shows the Five-year overall survival rate (OS) of the parotid MEC, and Figure two shows the Five-year survival rates of both the lymph node metastasis and parotid MEC.

Univariate analysis: As shown in Table one, factors such as clinical stage, lymph node metastasis, tumor size, surgical approach, distant metastasis, and pathological grade, etc., enabled to affect the prognosis of patients, but factors as age, gender, smoking and application of CGF in the surgical area appeared to have no significant influence on that.

Multivariate survival analysis: Multivariate analysis affecting prognosis was based on univariate Cox regression results, and incorporated clinical stage, lymph node metastasis, tumor size, surgical approach, distant metastasis, and pathological grade into further multivariate Cox regression analysis. The results indicated that lymph node metastasis, pathology Grade, and distant metastasis were independent factors affecting the prognosis of MEC of the parotid gland ( $p<0.01)$ as shown in Table two.

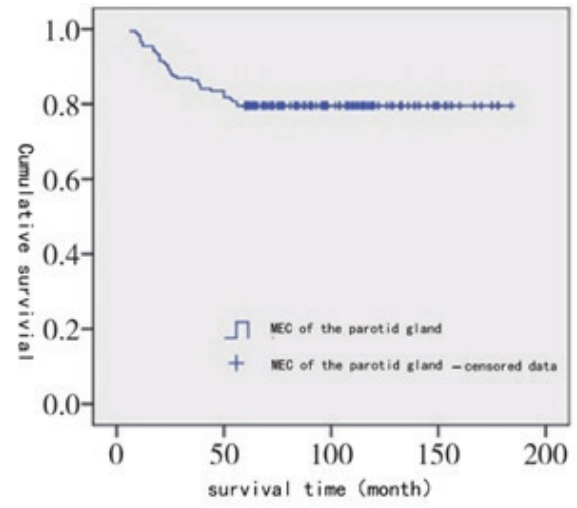

Figure 1 : Five-year overall survival rate (OS) of the

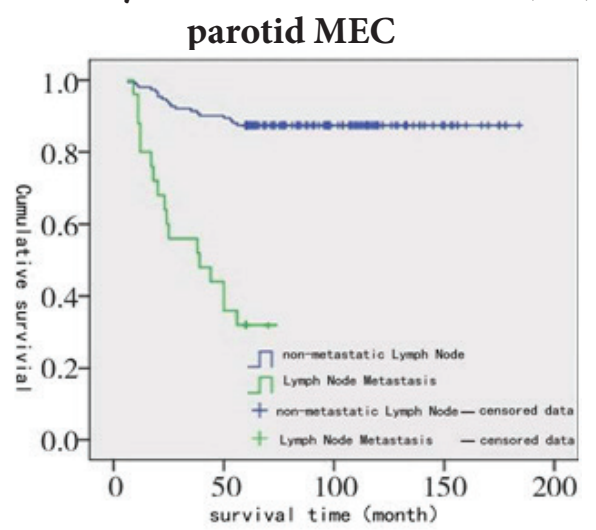

FIgure 2 : Five-year survival rates of both the lymph node metastasis and parotid MEC 


\section{BPKMCH}

Table 1: Univariate prognosis analysis of patients with mucoepidermoid carcinoma of the parotid gland

( $\mathrm{N}=176)$

\begin{tabular}{|c|c|c|c|c|c|c|c|c|c|}
\hline \multirow{2}{*}{ Factor } & \multirow{2}{*}{$\begin{array}{l}\text { Number } \\
\text { of case }\end{array}$} & \multirow{2}{*}{$\begin{array}{c}5 \text {-year } \\
\text { Survival } \\
\text { rate }(\%)\end{array}$} & \multirow{2}{*}{ p-value } & \multirow{2}{*}{ HR } & \multirow{2}{*}{$95 \% \mathrm{CI}$} & Factor & HR & $95 \% \mathrm{CI}$ & $\mathrm{p}$-value \\
\hline & & & & & & Clinical stage & 1.03 & $0.51-3.42$ & 0.68 \\
\hline Patient age (yrs.) & & & 0.23 & & & Lymph node metastasis & 5.52 & $2.85-12.05$ & $<0.01$ \\
\hline & 45 & 98 & 76.11 & 1 & & Tumor size & 1.36 & 0.62-3.09 & 0.43 \\
\hline & $\geq 45$ & 78 & 73.91 & 1.96 & $0.93-3.12$ & Therapy methods & 1.48 & $0.56-3.14$ & 0.93 \\
\hline Sex & & & 0.13 & & & Distant metastasis & 3.27 & $3.12-14.31$ & $<0.01$ \\
\hline Female & 84 & 77.38 & 1 & & & Pathological grade & 6.62 & $2.64-11.46$ & $<0.01$ \\
\hline
\end{tabular}

CGF

CGF Group

92

Control Group

\begin{tabular}{|l|c|c|c|c|c|}
\hline Clinical stage & & & 0.045 & & \\
\hline I & 29 & 100 & 1 & & \\
\hline
\end{tabular}

II

II

IV

Lymph node

metastasis

Negative

Postive

Tumor size

$\mathrm{T} 1$

$\mathrm{T} 2$

T3

T4

Smoking

Smoking group

Control group

Therapy Methods

Partial parotidectomy

Total

parotidectomy

\begin{tabular}{|l|c|c|c|c|c|}
\hline $\begin{array}{l}\text { Extended } \\
\text { parotidectomy }\end{array}$ & 45 & 58.74 & 4.48 & $2.48-2.9$ & \\
\hline $\begin{array}{l}\text { Combined radical } \\
\text { operation }\end{array}$ & 40 & 49.59 & 5.64 & $3.14-5.47$ & \\
\hline Distant metastasis & & & $<0.01$ & & \\
\hline Negative & 162 & 82.82 & 1 & & \\
\hline Positive & 14 & 14.28 & 10.37 & $5.1-18.72$ & \\
\hline Pathological grade & & & $<0.01$ & & \\
\hline Well & 77 & 92.39 & 1 & & \\
\hline Moderate & 65 & 81.27 & 1.31 & $0.52-1.53$ & \\
\hline Poor & 34 & 43.33 & 6.45 & $2.96-7.42$ & \\
\hline
\end{tabular}

NEPALESE JOURNAL OF CANCER (NJC)

Table 2: Multivariate prognostic analysis of patients with mucoepidermoid carcinoma of the parotid gland

$\mathrm{N}=176$

\section{Discussion}

Impact of gender and age on prognosis: Studies have shown that the prognosis of MEC of the parotid glands is different of different genders and ages. Choi et al. studied the survival factors of MEC after surgery and found that gender had an impact on the survival rate after surgery. ${ }^{5}$ The results of Roh et al. also proved that in the salivary MEC, the postoperative survival rate of male patients was significantly lower than that of female patients. ${ }^{6}$ Some scholars believed that age was closely related to the prognosis of MEC. The older the patient, the worse the prognosis. ${ }^{7}$ However, the results of this study showed that there was no correlation between age and gender and the prognosis of parotid MEC ( $\mathrm{p}>0.05)$.

Impact of surgical field application of CGF on prognosis: CGF, first extracted by Sacco in 2006, is a blood extract containing a variety of high-concentration growth factors. ${ }^{8}$ Studies have confirmed that CGF can promote the formation and regeneration of blood vessels and play an important regulatory role in the growth, remodeling, and repair of tissues. Meanwhile, CGF, derived from the body itself, with non-toxicity and immunogenicity, has no immune rejection. ${ }^{9}$ According to observation by clinical dressing change, on the 3rd day after surgery, the patients who applied CGF during the operation had clean wounds, more granulation tissue growth, and less inflammation around the wound. However, the traditional surgery did not smear the redness and swelling around the operation area, and the inflammation was obvious. Ten days later after the surgery, the wounds of patients with CGF in the operation area healed, and the scars were not obvious. On the 14th post-operative day, the patients who had been treated 
with traditional surgery without CGF healed and there was obvious scar formation. This study showed that the application of CGF in the surgical area was irrelevant to the prognosis of MEC $(p=0.67)$, but the application of CGF in the surgical area could promote healing of the surgical area and reduce postoperative scars. Moreover, CGF, extracted from autologous venous blood, was promoted for clinical use for its simple manufacturing process, high safety, and low cost.

Impact of clinical staging on prognosis: Most scholars argued that the clinical stage as one of the important factors affecting the prognosis of patients with parotid MEC. LR et al. pointed out that for patients in clinical stage III and IV parotid MEC, the tumor diameter was large or regional lymph node metastasis exists, and when there was lymph node metastasis before surgery, the postoperative distant organ metastasis was easier. ${ }^{10}$ The report from Plambeck et al. among 52 patients of salivary MEC who received surgical treatment, found that all the fatal cases were dead in their clinical stage III or IV. Meanwhile, Hicks et al. detected that the survival rate of patients with clinical stage III and IV parotid MEC significantly decreased than those of stages I and II. The results show that the clinical stage was closely related to postoperative survival. Patients with stage I and II parotid MEC had higher postoperative survival rates than those with stage III and IV $(\mathrm{p}<0.05)$. The results were consistent with those reported in the literature.

Impact of lymph node metastasis on prognosis: There was no accordant conclusion about the impact of cervical lymph node metastasis on prognosis. Liu et al. ${ }^{11}$ counted 9 independent studies on the prognosis of salivary glands, and 7 of them reported that cervical lymph node metastasis was associated with poor prognosis in univariate analysis. But in a multivariate analysis, none of the six reports showed it as an independent prognostic factor. However, Chen et al. ${ }^{12}$ supported lymphatic metastasis as an independent poor prognostic factor ( $\mathrm{p}$ $<0.01$ ) based on a study of 2,400 patients with salivary MECs. In this study, 25 of 176 patients had lymph node metastasis, with a metastasis rate of $14.2 \%$. The Five-year survival rate of patients with lymph node metastasis in the mucoepidermoid carcinoma of the parotid gland was significantly lower than that in the non-metastatic lymph node group, which was statistically significant $(\mathrm{p}<0.05)$. The results demonstrated that lymph node metastasis was an adverse factor for recurrence and metastasis of patients with MEC in the salivary gland, which were consistent with the conclusions of Chen et al. ${ }^{11}$, thus cervical lymph node dissection should be actively performed for patients who meet the indications.

Impact of tumor size on prognosis: For MEC, as the tumor grew, the prognosis got worse, and the course of the disease developed as the tumor volume increased. Studies had shown that a malignantly transformed cell counted for an increase of about 40 cell cycles and reached1012 tumor cells, which can cause extensive metastases. ${ }^{13}$ The results of this study indicated that the Five-year survival rate for patients with stage T1 is 100\% (27/27). The Five-year survival rate of patients with the T4 stage was only $55.17 \%$ (32/58). Univariate analysis showed a statistically significant difference in the effect of tumor size on prognosis $(\mathrm{p}<0.05)$. The larger the tumor volume, the worse the prognosis.

Impact of smoking on prognosis: A large number of literatures reported that smoking is one of the risk factors for the recurrence of benign tumors of the parotid gland, such as Warthin tumor and mixed tumor of the parotid gland. ${ }^{14}$ This study shows that the Five-year survival rate of patients in the smoking group is lower than that in the non-smoker group, but the difference has no statistical significance.

Impact of surgical methods on prognosis: The thoroughness of the first operation is also a key factor affecting the prognosis. One hundred seventy-six patients of this group were treated with surgery for the first time, consisting of four surgical methods: Partial parotidectomy, Total parotidectomy, Extended parotidectomy, and Combined radical operation. According to the univariate analysis of the effects of the four procedures on the prognosis, the difference had a statistical significance. With the expansion of the scope of surgery, the Five-year survival rate had shown a downward trend, which was related to the proportion of patients' clinical stage. In the partial parotidectomy group, T3 and T4 patients accounted for only $20.37 \%$ $(11 / 54)$, while in the combined radical operation group, 
T3 and T4 patients accounted for 72.5\% (29/40). Spiro et al. ${ }^{15}$ believed that for patients with clinical stage I and II, a pathological grade of highly differentiated and insignificant symptoms, simple lesion resection could be used, and Combined radical surgery was generally not recommended unless they had the clinically enlarged lymph nodes to be suspected of metastasis. In the special parotid gland MEC, if there are no facial nerve symptoms, a parotid or subtotal resection of the facial nerve should be performed. The author's view was consistent with it.

Impact of distant metastasis on prognosis: The incidence rate of distant metastasis in MEC was relatively low. ${ }^{16}$ Only 14 cases of 176 patients in this group were present with distant metastasis. The univariate analysis showed that the 5-year survival rates of non-distant metastasis and distant metastasis respectively were $82.82 \%$ and $14.28 \%$. The difference between both categories was statistically significant $(\mathrm{p}<0.05)$. The multivariate analysis showed that distant metastasis was an independent factor affecting prognosis. MEC's insensitivity to chemotherapy was the main reason for failure to treat distant metastases. Jun-Zheng $\mathrm{Wu}$ and others in the study of anti-tumor drug sensitivity of mucoepidermoid cancer cells found that they were generally sensitive to fluorouracil, vinblastine, retinoic acid, and interferon, ${ }^{17}$ but clinical studies on MEC chemotherapy are still insufficient in research.

Impact of pathological grading on prognosis: Histologically, squamous cell carcinoma is classified into three levels: highly differentiated, moderately differentiated and poorly differentiated, according to the degree of keratinization of the malignant tumor tissue, the nuclear polymorphism of cells, and the activity of mitosis. ${ }^{18}$ A large number of studies had shown that pathological grading of MEC had an impact on both tumor-free survival and overall survival rate after surgery, and as one of the risk factors for postoperative metastasis. ${ }^{19}$ This study indicated that the pathological grading of the parotid gland affected the prognosis of MEC, as an independent factor affecting its prognosis. Thus, the poorly differentiated tumor trended to have a worse prognosis. However, because MEC cell differentiation is a continuous process, and the degree of differentiation is considered to be defined according to the cytological morphology, which is prone to certain errors. In the clinical Bone field, it still existed that highly differentiated MECs trended to have an infiltrative growth and be more aggressive to surrounding tissues and prone to recurrence and metastases. Therefore, clarifying the pathological grade of the tumor cannot only indicate the prognosis but also guide the treatment. As for poorly differentiated tumors, the scope of resection should be expanded, combined with other factors (such as a late clinical-stage), and regional cervical lymph node dissection or postoperative radiotherapy should be properly taken to improve the cure rate.

\section{Conclusion}

This study indicated that the clinical stage, lymph node metastasis, tumor size, surgical method, distant metastasis, and pathological grading affected the prognosis of patients. Lymph node metastasis, pathological grade, and distant metastasis were independent factors affecting the prognosis of the parotid MEC. The worse the tumor differentiation, the worse the prognosis for patients with lymph nodes and distant metastases. Due to the less sample size in the retrospective analysis, there might be bias. In the future, the larger sample size is needed to further evaluate the impact of clinical-pathological factors and treatment methods on the prognosis of parotid MEC.

In summary, the results indicated that the overall prognosis of the parotid MEC was good. In the treatment of parotid MEC, facial glands-reserving glands would help improve the tumor prognosis. For patients with lymph node metastasis, distant metastasis, and poorly differentiated tumors, the prognosis was significantly worse than other pathologies. How to improve the therapeutic effect of such patients is a main researching direction in the future.

\section{References}

1. Chao Li. Status and prospects of multidisciplinary comprehensive treatment of head and neck tumors. Journal Of Cancer Control And Treatment. 2014;27( 4):161-164.

2. McHugh JB, Visscher DW, Barnes EL. Update on selected salivary gland neoplasms. Arch Pathol Lab Med. 2009; 133( 11): 1763-1774. 
3. Wen J, Li HT, Li SH, Li X, Duan JM. Investigation of modified platelet-rich plasma (mPRP) in promoting the proliferation and differentiation of dental pulp stem cells from deciduous teeth.Braz J Med Biol Res. 2016;49(10):e5373.

4. Nguyen TH, Palankar R, Bui VC, Medvedev N, Greinacher A, Delcea M. Rupture forces among human blood platelets at different degrees of activation. Sci Rep. 2016; 5(6): 25402.

5. Choi G, Jung K Y, et a1, Low expression of p27(Kipl) in advanced mucoepidermoid carcinomas of head and neck[J]. Head Neck. 2001; 23(4): 292-297.

6. Roh J, Muelleman T, Tawfik O, et al. Perineural growth in head and neck squamous

7. Chen AM, Lau VH, Farwell DG, et al. Mucoepidermoid carcinoma of the parotid gland treated by surgery and postoperative radiation therapy: clinicopathologic correlates of outcome [J]. Laryngoscope. 2013; 123( 12):3049-3055.

8. Kreuz PC, Krüger JP, Metzlaff S, Freymann U, Endres M, Pruss A, Petersen W, Kaps C. Platelet-rich plasma preparation types show impact on chondrogenic differentiation, migration, and proliferation of human subchondral mesenchymal progenitor Cells. Arthroscopy. 2015;31(10):1951-1961.

9. Yu B, Wang Z. Effect of concentrated growth factors on beagle periodontal ligament stem cells in vitro.[J] Mol Med Rep. 2014; 9 (1): 235-242.

10. Rhee CS, Won TB, Lee CH, et al. Adenoid cystic carcinoma of the sinonasal tract: treatment results[J]. Laryngoscope. 2006; 116(6):982-986.

11. Liu S, zOw A, Ruan M, et al. Prognostic factors in primary salivary gland mucoepidermoid carcinoma: an analysis of 376 cases in an Eastern Chinese population. Int J Oral Maxillofac Surg. 2014; 43 (6):667-673.

12. Chen MM, Roman SA, Sosa JA, et al. Histologic grade as prognostic indicator for mucoepidermoid carcinoma: a population-level analysis of 2400 patients [J]. Head Neck. 2014; 36( 2):158-163.

13. NozoeT, EzakiT, Kabashima A, et al. Significance of immunohistochemical expression of cyclooxygenase- 2 in squamous cell carcinoma of the esophagus [J]. Am J Surg. 2005; 189(1): 110-115.

14. Shin YS, Koh YW, Kim SH, et al. Selective neck dissection for clinically node-positive oral cavity squamous cell carcinoma [J].Yonsei Med J. 2013; 54(1): 139-144.

15. Sprio I J, Wang C C, Montgomery W W, Carcinoma of the parotid gland.Analysis of treatment results and patterns of failure after combined surgery and radiation therapy[J].Cancer. 2011; 71(9): 2699-2705.

16. Xi-Ze Zhang, Wei-Liu Qiu, Oral and Maxillofacial Surgery[M]. Beijing People's Medical Publishing House. 1987: 290- 291.

17. Zheng-Jun WU, Jian-Yuan Chen, Characteristics of drug sensitivity of salivary gland mucoepidermoid carcinoma with high metastatic cells. Journal of Practical Stomatology. 1997; 13(2): 118-120.

18. Ding D,Stokes W,Eguchi M,et al. Association between lymph node ratio and recurrence and survival outcomes in patients with oral cancer. JAMA Otolaryngol Head Neck Surg. 2018; 145(1): 53-61.

19. Triantafillidou K, Dimitrakopoulos J, Iordanidis F, et al. Mucoepidermoid carcinoma of minor salivary glands: a clinical study of 16 cases and review of the literature[J].Oral Dis. 2006; 12(4):364-370. 